\title{
Reconfiguration of Three Phase MAF-SRF-PLL as Single Phase PLL
}

\author{
Anirban Ghoshal ${ }^{1} \cdot \operatorname{Vinod}$ John $^{2}$
}

Received: 5 July 2016/Accepted: 10 July 2016/Published online: 19 July 2016

(C) Indian National Academy of Engineering 2016

\begin{abstract}
Improved SRF-PLL using moving average filter (MAF) is an established phase locked loop method for operation of three phase grid connected VSI under nonideal grid conditions. This three phase PLL method can also be used in a single phase system without changing its structure. Hence, in applications where the grid connected VSI has to work under both three phase and single phase source, a MAF-SRF-PLL is useful for maintaining proper synchronism. In this letter, first an already available design of three phase MAF-SRF-PLL has been evaluated experimentally under various single phase grid condition in terms of unit vector quality. Next the same PLL structure has been tested for situations where circuit reconfiguration from three phase to single phase and vice versa are necessary.
\end{abstract}

Keywords Phase locked loop - Synchronous reference frame - Moving average filter - Frequency estimation . Grid connected inverter

\section{Introduction}

Synchronous reference frame (SRF) PLL is an established PLL method for three phase grid with near ideal operating conditions (Nicastri and Nagliero 2010; Kulkarni and John 2013). Several techniques are present in literature to improve its performance under non-ideal grid condition such as unbalance (Nicastri and Nagliero 2010; Golestan

Anirban Ghoshal

ghoshal.anirban@gmail.com

Midnapore, India

2 Department of EE, IISc, Bangalore, India et al. 2014). Use of moving average filter (MAF) (Golestan et al. 2014, 2015; Ghoshal and John 2007; Mirhosseini et al. 2014) inside the closed loop SRF-PLL is one such technique. A MAF-SRF-PLL has been shown to operate under several non-ideal grid condition including extreme unbalance situation such as single phase line to ground fault condition (Golestan et al. 2014; Ghoshal and John 2007). For a three phase SRF-PLL the three phase to two phase transformation generates two $90^{\circ}$ phase separated signals. In a single phase system the main issue is generation of a $90^{\circ}$ phase shifted signal to that of the available phase voltage (Golestan et al. 2013). In general the available methods of single phase PLL use additional filter structure to produce a $90^{\circ}$ phase shifted signal from input phase voltage and then use them as ' $\alpha$ ' and ' $\beta$ ' component of voltages in a SRF-PLL (Golestan et al. 2013). Considering single phase system as a special case of three phase unbalanced system, a three phase MAF-SRF-PLL structure can be used to generate unit vectors synchronised to single phase grid voltage (Mirhosseini et al. 2014). Therefore in reliability critical applications where a grid connected VSI needs to operate under three phase balanced, three phase unbalanced and single phase condition, a MAF-SRF-PLL can be used to maintain synchronism with electric grid. Example of such a necessity are present in VSIs operating with high reliability drawing unity power factor current from the grid such as telecommunication power system and electric transportation system. A phase selection algorithm should be present along with PLL to determine different operating conditions as shown in Fig. 1.

In this work an already available design of three phase MAF-SRF-PLL, presented in Ghoshal and John (2007), has been chosen and evaluated with a single phase source. Necessary theoretical understandings have been developed by taking single phase system as an unbalanced three phase 


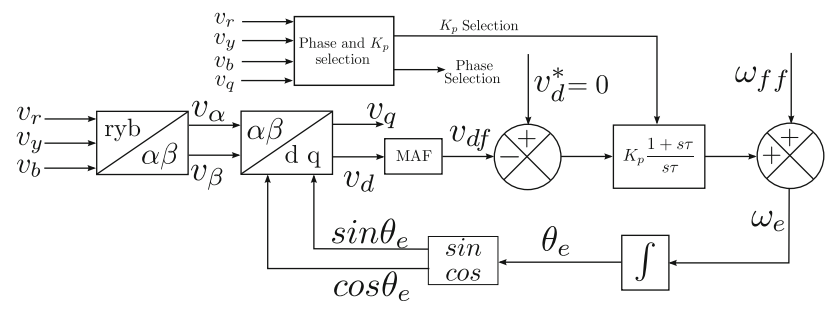

Fig. 1 Block diagram of MAF-SRF-PLL

system. The performance of the PLL has been evaluated in terms of unit vector Total Harmonic Distortion (THD) (Kulkarni and John 2013) and settling time. Experimental results for various conditions in a single phase grid and for reconfiguration between three phase and single phase grid are presented.

\section{Single Phase MAF-SRF-PLL}

Block diagram of a three phase MAF-SRF-PLL is shown in Fig. 1. Such a MAF-SRF-PLL can also be used for a single phase system but without the necessity of an exclusive method for quadrature component generation. A single phase system can be considered as an unbalanced three phase system. The sequence voltages can be derived by carrying out a sequence decomposition. The expressions for sequence voltage for this case in two phase stationary $\alpha \beta$ domain can be derived as shown in Eqs. (1) and (2)

$v_{\alpha p}=\frac{V_{m}}{2} \cos (\omega t) \quad$ and $\quad v_{\alpha n}=\frac{V_{m}}{2} \cos (\omega t)$

$v_{\beta p}=\frac{V_{m}}{2} \sin (\omega t) \quad$ and $\quad v_{\beta n}=-\frac{V_{m}}{2} \sin (\omega t)$

For a single phase condition $v_{\alpha}=v_{r}$ and $v_{\beta}=0$, which can also be understood from Eqs. (1) and (2). Using estimated phase angle $\theta_{e}, \mathrm{~d}$ and $\mathrm{q}$ axis voltages are calculated. The method applied to counter unbalanced condition by incorporating a MAF structure can be extended to this situation also. The use of MAF would attenuate the ripple component present in $v_{d}$ and thereby achieving correct estimation of $\theta_{e}$. The $d$ and $q$ axis voltage can be calculated at steady state by using $\alpha \beta$ domain voltages. Assuming that only positive sequence phase angle is present in estimated angle $\theta_{e}$, the $\mathrm{d}$ and $\mathrm{q}$-axis voltages at steady state can be expressed as given in Eqs. (3) and (4).

$v_{d}=\frac{V_{m}}{2} \sin (2 \omega t)$

$v_{q}=\frac{V_{m}}{2}+\frac{V_{m}}{2} \cos (2 \omega t)$

The gain of PI controller is dependent on the magnitude of voltage space vector, which can be observed from block

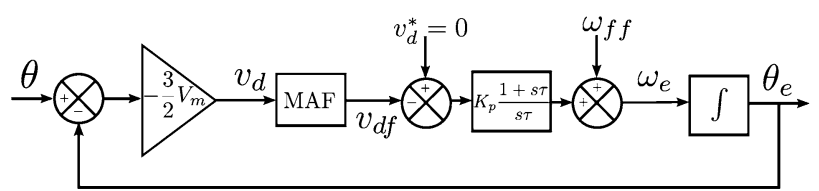

Fig. 2 Simplified closed loop block diagram of MAF-SRF-PLL

diagram of Fig. 2. It can be derived that the linearised three phase SRF-PLL has a gain block of $-\frac{3}{2} V_{m}$ and during unbalance it changes to a gain of $-\frac{3}{2} V_{m p}$, where $V_{m p}$ is positive sequence peak fundamental voltage (Ghoshal and John 2007). For a single phase system the sequence voltage analysis show that peak of positive sequence fundamental voltage in the $r y b$ reference frame is $V_{m p}=\frac{V_{m}}{3}$. Hence, effective gain for a single phase PLL would be $-\frac{V_{m}}{2}$. Therefore to tune the system properly for a single phase case, PI gains need to be calculated using this gain factor of $-\frac{V_{m}}{2}$. However the calculation procedure remains same for both three phase and single phase systems.

\section{Operating Conditions}

The three phase MAF-SRF-PLL structure chosen here is same for both the three phase and single phase system. The two operating conditions are given below.

Case-1: a single phase system only.

Case-2: either three phase or single phase system

For the first case the supply is a single phase system. The three phase PLL input $v_{r}$ would equal to $v_{\alpha}$ and $v_{\beta}=0$. In this case the three phase to two phase transformation can be avoided and the input voltage can directly be given as $v_{\alpha}$ while making $v_{\beta}=0$.

In the second case the same PLL structure is required to operate either with a three phase or a single phase system. Therefore its input needs to be reconfigured during switch over between three phase and single phase. For example if the ' $\mathrm{B}$ ' phase supply is suddenly disconnected then a loss of phase detection would change the PLL inputs to $v_{r}=v_{R Y}, v_{y}=0$ and $v_{b}=0$. Where $v_{R Y}$ is the line to line voltage between remaining healthy ' $R$ ' and ' $Y$ ' phase. Here a best phase selection algorithm monitors the grid voltage condition and selects proper phases for power converter operation.

\section{Experimental Result}

The theoretical idea developed in "Introduction" on $1-\phi$ MAF-SRF-PLL is experimentally verified by implementing it on a Altera Cyclone-II series FPGA board. The 
computations have been carried out in fixed point arithmetic method with a register size of 16 bit. The clock frequency of the FPGA has been set at $20 \mathrm{MHz}$ and the total execution time of the single phase MAF-SRF-PLL has been $2.5 \mu$ s. Settling time and quality of produced unit vectors in terms of THD have been chosen as performance assessment indices. Various grid voltage situations have been created by using a laboratory made grid simulator. The tabulated values of THD of both the unit vectors, $\sin \theta_{e}$ and $\cos \theta_{e}$, are calculated from the stored data using a digital oscilloscope. An off-line frequency spectrum

Table 1 Performance of MAF-SRF-PLL with $1-\phi$ supply

\begin{tabular}{lllll}
\hline $\begin{array}{l}\text { Supply } \\
\text { frequency } \\
(\mathrm{Hz})\end{array}$ & $\begin{array}{l}\text { Harmonic } \\
\text { in grid } \\
\text { voltage }\end{array}$ & \multicolumn{2}{l}{ THD of unit vector $(\%)$} & $\begin{array}{l}\text { Settling } \\
\text { time }(\mathrm{ms})\end{array}$ \\
\cline { 2 - 4 } & $\operatorname{Cos} \theta_{e}$ & $\operatorname{Sin} \theta_{e}$ & \\
\hline 50 & Not present & 1.06 & 1.04 & 50 \\
47.5 & Not present & 1.10 & 0.95 & 50 \\
50 & Present & 1.21 & 1.24 & 50 \\
47.5 & Present & 1.32 & 1.24 & 50 \\
\hline
\end{tabular}

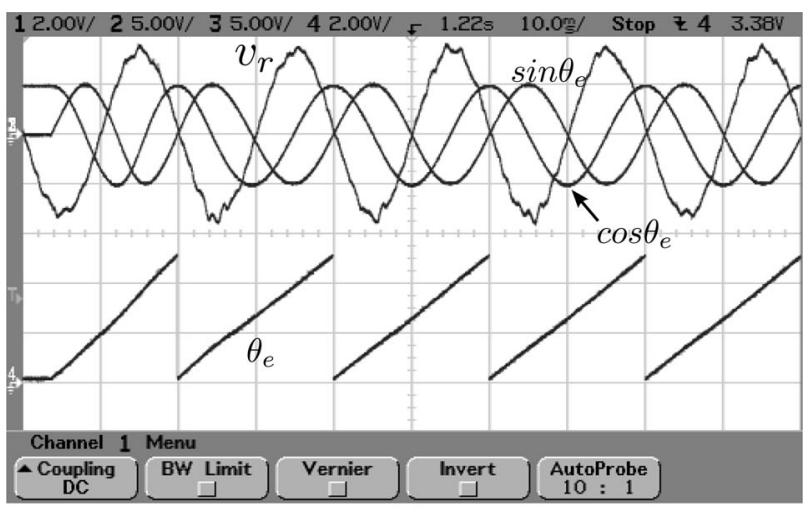

(a)

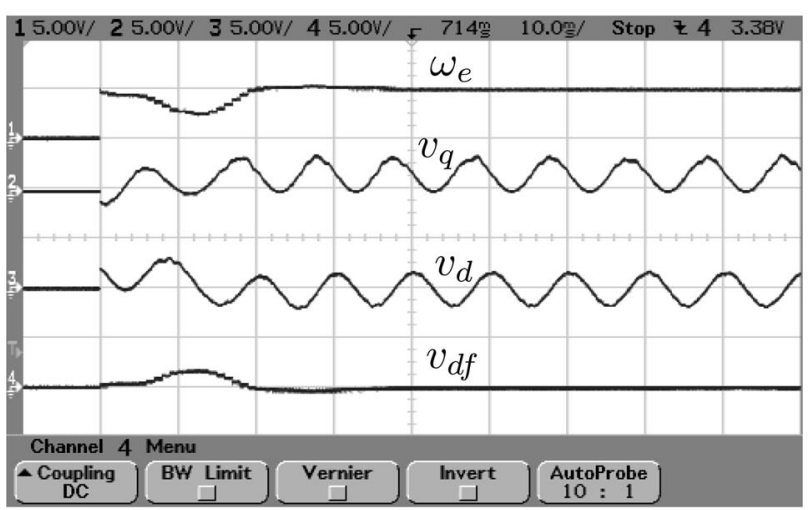

(b)

Fig. 3 Start-up transient and steady state operation of MAF-SRFPLL with single phase supply. a Input voltage $v_{r}$, estimated phase $\theta_{e}$ and unit vectors $\sin \theta_{e}$ and $\cos \theta_{e}$ at frequency of $50 \mathrm{~Hz}$. b Estimated frequency $\omega_{e}, \mathrm{~d}$ axis voltage $v_{d}$, q axis voltage $v_{q}$ and moving average filtered d axis voltage $v_{d f}$ at frequency of $50 \mathrm{~Hz}$ analysis have been carried out on each set of data and from which THD of unit vectors have been calculated and listed in Table 1. The experimental results are discussed below.u

Case-1: start up and steady state performance of the three phase MAF-SRF-PLL under single phase grid condition has been experimentally tested using the laboratory made grid voltage simulator. For single phase source with supply frequency of $50 \mathrm{~Hz}, \theta_{e}, \sin \theta_{e}, \cos \theta_{e}$ and phase voltage $v_{r}$ under both start-up and steady state condition are shown in Fig. 3a. In Fig. $3 \mathrm{~b} \omega_{e}, v_{d}, v_{q}$ and $v_{d f}$ are shown. $v_{d}$ and $v_{q}$ contain $100 \mathrm{~Hz}$ ripple as expected and the filtered output of the MAF, $v_{d f}$, settles to zero because of high attenuation at $100 \mathrm{~Hz}$. It can be observed that $\sin \theta_{e}$ is at $180^{\circ}$ phase shift with $v_{r}$, which is an indication of phase locked situation. Performance of PLL with harmonic rich input at $50 \mathrm{~Hz}$ and single phase source with distorted voltage waveform at $47.5 \mathrm{~Hz}$ are shown in Fig.4a, b. The quality of unit vectors in terms of THD is tabulated in Table 1 and the values are well within $2, \%$ for all the above described cases. PLL settling time for these situations are nearly $50 \mathrm{~ms}$.

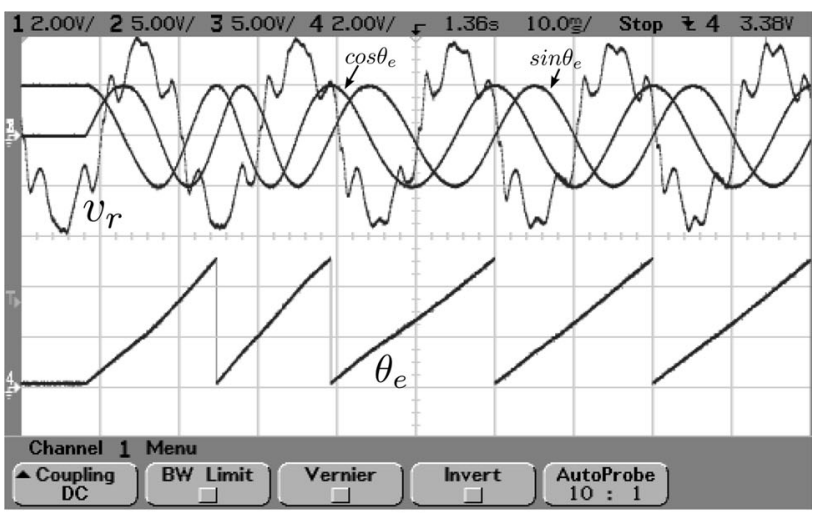

(a)

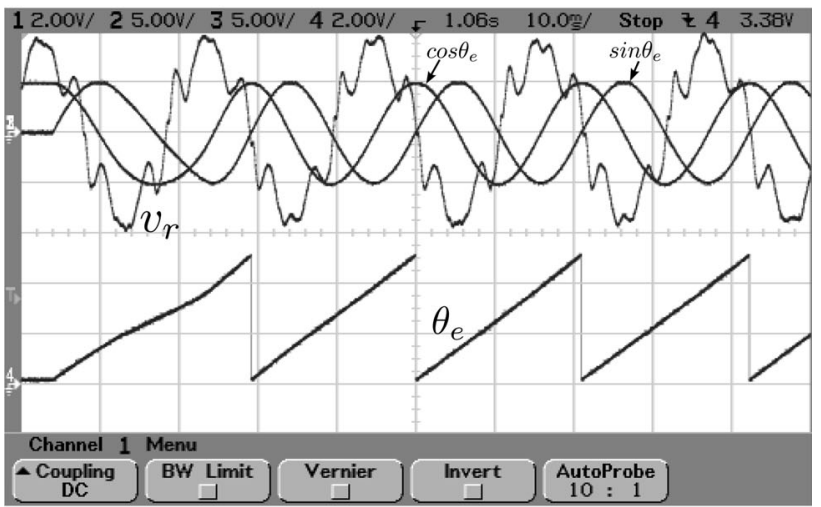

(b)

Fig. 4 Phase tracking by MAF-SRF-PLL with distorted input voltage. a Input voltage frequency $50 \mathrm{~Hz}$. b Input voltage frequency $47.5 \mathrm{~Hz}$ 


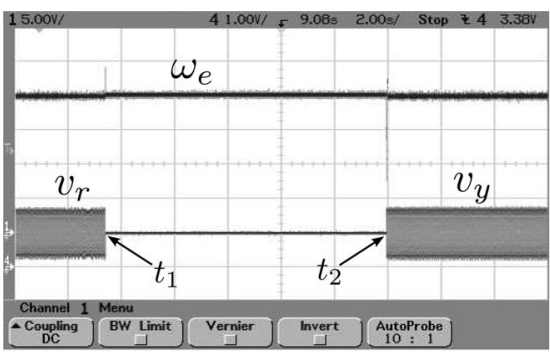

(a)

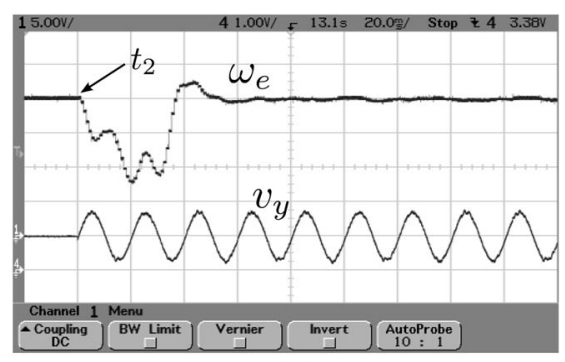

(b)

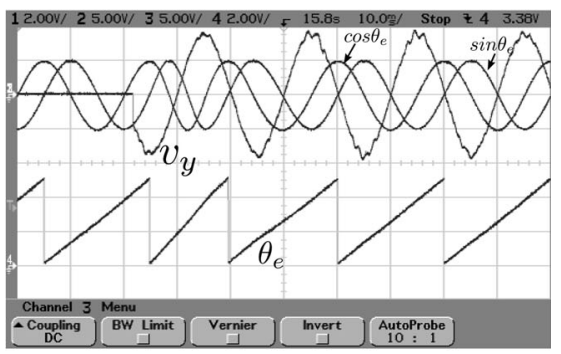

(c)

Fig. 5 Operation of $1-\Phi$ MAF-SRF-PLL during phase change. Operation of $1-\Phi$ MAF-SRF-PLL during phase change. a Frequency estimation, $\mathbf{b}$ frequency estimation at the point of recovery and $\mathbf{c}$ phase angle estimation

A single phase front end converter may see a loss of supply and reappearance of supply from a different phase after few seconds, such as in traction system. In Fig. 5a such an input supply condition is shown along with the estimated frequency $\omega_{e}$ by PLL. During loss of input to PLL it is possible for the estimated frequency to drift away from nominal frequency due to integrator of PI controller of the PLL and settle to upper bound or lower bound decided by its upper or lower limit. To restrict $\omega_{e}$ from drifting away a signal indicating loss of supply from $v_{q}$ has been generated and is used to stop the PI controller integrator of PLL. For this, $v_{q}$ is filtered with a similar MAF structure used for $v_{d}$ and loss of supply is indicated if filtered value of $v_{q}$ falls below a specified value. The output of the MAF settles within $10 \mathrm{~ms}$ so loss of supply can be detected well within half a cycle of fundamental frequency. Whenever there is a loss of supply, $\omega_{e}$ is held at its current value, shown in Fig. 5a. After reappearance of supply the integrator is released within $10 \mathrm{~ms}$ and the PLL resumes normal operation. During reappearance of supply from Y phase at $t_{2}$ the transient takes about $60 \mathrm{~ms}$ to settle, shown in Fig. 5b. The unit vector generation, Fig. 5c, show that the PLL structure is able to phase lock with the grid after reappearance of supply.

Case-2: for the situation when a power converter is required to reconfigure itself from operation under three phase to single phase and vice versa, the experimental results on maintaining synchronisation with input voltage using a three phase MAF-SRF-PLL are shown in Fig. 6. Initially all the three phase voltages are present and hence $\theta_{e}$ is in locked condition with ' $\mathrm{R}$ ' phase voltage $v_{r}$, shown in Fig. 6a. Due to sudden disconnection of ' $\mathrm{B}$ ' phase voltage, the PLL is now required to synchronise with line to line voltage $v_{R Y}$. Here line voltage $v_{R Y}$ is the available single phase grid voltage to the PLL and power converter. It can be observed from Fig. 6a that ' $\theta_{e}$ ' becomes locked to $v_{R Y}$ after the change over and $\omega_{e}$ reaches steady state within three cycles. The reverse process that is the change over from single phase input $v_{R Y}$ to three phase input is also shown in Fig. $6 \mathrm{~b}$. These results show that the three phase MAF-SRF-PLL can successfully be used in a situation when a power converter needs to reconfigure itself for operation both under three phase and single phase supply in application where high level of reliability and power converter availability is required.

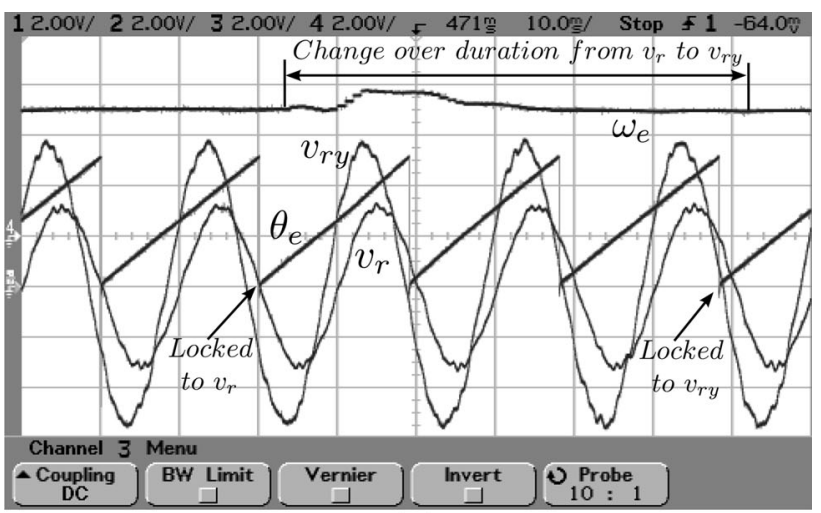

(a)

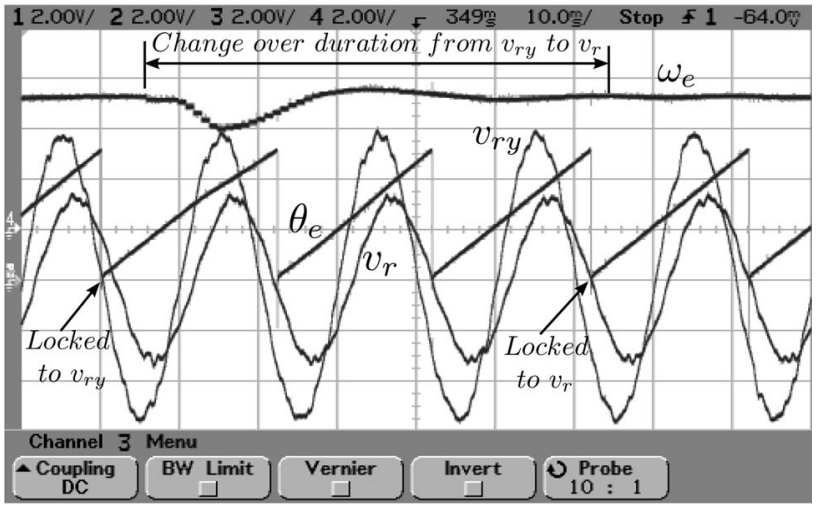

(b)

Fig. 6 Phase tracking during reconfiguration from a three phase to single phase (line to line) and $\mathbf{b}$ single phase (line to line) to three phase systems 


\section{Conclusion}

In this work capability of a three phase MAF-SRF-PLL has been experimentally verified to work under a single phase source without altering its structure. The experimental result confirms the ability of a three phase MAF-SRF-PLL to work as a single phase PLL under various non-ideal grid conditions. The performance evaluation of the MAF-SRFPLL under single phase source show, that it can produce unit vectors with THD well within ' $2 \%$ ' and has a settling time of $50 \mathrm{~ms}$. The structure and design procedure of this MAF-SRF-PLL remains same under both single phase and three phase conditions. Experimental results show its ability to maintain synchronism with electric grid even if there is a supply phase change or if the system needs to reconfigure itself from operating under three phase to single phase supply or vice-versa.

\section{References}

Nicastri A, Nagliero A (2010) Comparison and evaluation of the PLL techniques for the design of the grid-connected inverter systems. IEEE Int. Symp. on Ind. Electronics (ISIE), pp 3865-3870
Kulkarni A, John V (2013) Analysis of bandwidth-unit vector distortion trade off in PLL during abnormal grid conditions. IEEE Trans Ind Electron 60(12):5820-5829

Golestan S, Ramezani M, Guerrero JM, Francisco DF, Monfared M (2014) Moving average filter based phase-locked loops: performance analysis and design guidelines. IEEE Trans Power Electron 29(6):2750-2763

Ghoshal A, John V (2007) A method to improve PLL performance under abnormal grid conditions. Proc. National Power Electronics Conference, Bangalore, India

Golestan S, Ramezani M, Guerrero JM, Monfared M (2015) dqFrame cascaded delayed signal cancellation- based PLL: analysis, design, and comparison with moving average filter-based PLL. IEEE Trans Power Electron 30(3):1618-1632

Mirhosseini M, Pou J, Agelidis VG, Robles E, Ceballos S (2014) A three-phase frequency-adaptive phase-locked loop for independent single-phase operation. IEEE Trans Power Electron 29(12):6255-6259

Golestan S, Monfared M, Freijedo FD, Guerrero JM (2013) Dynamics assessment of advanced single-phase PLL structures. IEEE Trans Ind Electron 60(6):2167-2177 